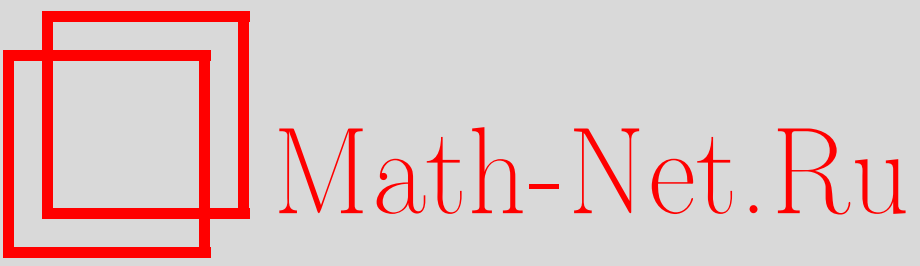

С. Е. Конштейн, 3-частичная модель Калоджеро: суперследы и идеалы на алгебре наблюдаемых, ТМФ, 1998, том 116, номер 1, 122-133

DOI: https://doi.org/10.4213/tmf892

Использование Общероссийского математического портала Math-Net.Ru подразумевает, что вы прочитали и согласны с пользовательским соглашением

http://www.mathnet.ru/rus/agreement

Параметры загрузки:

IP : 18.208 .226 .222

26 апреля 2023 г., 14:57:04

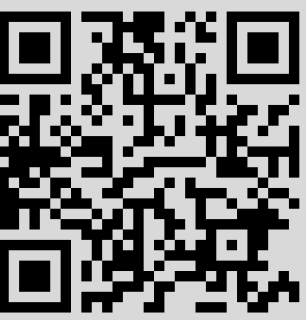


ТЕОРЕТИЧЕСКАЯ

И МАТЕМАТИЧЕСКАЯ

ФИЗИКА

Том 116, № 1

июль, 1998

(C) 1998 г.

С. Е. Конштейн*

\section{3-ЧАСТИЧНАЯ МОДЕЛЬ КАЛОДЖЕРО: СУПЕРСЛЕДЫ И ИДЕАЛЫ НА АЛГЕБРЕ НАБЛЮДАЕМЫХ}

Ассоциативная супералгебра наблюдаемых 3 -частичной квантовой модели Калоджеро, позволяющая при помощи стандартной фоковской процедуры получить все волновые функции модели, имеет два независимых суперследа. Показано, что при константе связи $\nu$, имеющей вид $\nu=n \pm 1 / 3$ или $\nu=n+1 / 2$, наличие суперследов ведет к существованию в этой супералгебре нетривиальных двусторонних идеалов.

\section{1. ВВЕДЕНИЕ}

Трехчастичная модель Калоджеро [1] является удобным объектом для изучения некоторых свойств $N$-частичных моделей Калоджеро, т.к. она уже имеет черты, отличающие многомерные системы от одномерной, но еше остается легко решаемой задачей, допускающей разделение переменных [2].

Хорошо известно, что с помошью преобразования подобия гамильтониан модели Калоджеро может быть сведен к оператору

$$
H_{\mathrm{Cal}}=-\frac{1}{2} \sum_{i=1}^{N}\left[\frac{\partial^{2}}{\partial x_{i}^{2}}-x_{i}^{2}+\nu \sum_{j \neq i} \frac{2}{x_{i}-x_{j}} \frac{\partial}{\partial x_{i}}\right],
$$

который на пространстве симметрических функций совпадает с оператором

$$
H=\frac{1}{2} \sum_{i=1}^{N}\left\{a_{i}^{0}, a_{i}^{1}\right\}
$$

В этом выражении операторы рождения и уничтожения $a^{\alpha}(\alpha=0,1)[3,4]$ выражаются через дифференциально-разностные операторы Данкла $D_{i}(x)[5]$

$$
a_{i}^{\alpha}=\frac{1}{\sqrt{2}}\left(x_{i}+(-1)^{\alpha} D_{i}(x)\right),
$$

* Отделение теоретической физики им. И. Е. Тамма Физического института им. П.Н. Лебедева РАН, Москва, Россия. Е-mail: konstein@td.lpi.ac.ru 
где

$$
D_{i}=\frac{\partial}{\partial x_{i}}+\nu \sum_{l \neq i}^{N} \frac{1}{x_{i}-x_{l}}\left(1-K_{i l}\right)
$$

а $K_{i j}=K_{j i}$-операторы элементарных перестановок индексов

$$
K_{i j} x_{i}=x_{j} K_{i j}, \quad K_{i j} x_{k}=x_{k} K_{i j} \text { при } k \neq i, \quad k \neq j .
$$

Операторы $a^{\alpha}$ удовлетворяют коммутационным соотношениям

$$
\left[a_{i}^{\alpha}, a_{j}^{\beta}\right]=\epsilon^{\alpha \beta}\left(\delta_{i j}+\nu \delta_{i j} \sum_{l=1, l \neq i}^{N} K_{i l}-\nu \delta_{i \neq j} K_{i j}\right)
$$

где $\epsilon^{\alpha \beta}=-\epsilon^{\beta \alpha}, \epsilon^{01}=1$, из которых следует [4], что операторы (3) удовлетворяют соотношениям

$$
\left[H, a_{i}^{\alpha}\right]=-(-1)^{\alpha} a_{i}^{\alpha}
$$

и поэтому операторы $a_{i}^{1}$, действуя на фоковский вакуум $|0\rangle$ такой, что $a_{j}^{0}|0\rangle=0$ для всех $j$, дают волновые функции модели (2), а значит, и модели (1). В [6] операторы Переломова [7], описываюшие волновые функции модели (1), выражены с помошью этих операторов $a_{i}^{\alpha}$.

Далее алгеброй наблюдаемьх будет называться алгебра $S H_{N}(\nu)$ - ассоциативная супералгебра полиномов от операторов $a_{i}^{\alpha}(3)$ и $K_{i j}$, в которой $\mathbb{Z}_{2}$-градуировка задается четностью $\pi: \pi\left(a_{i}^{\alpha}\right)=1, \pi(1)=\pi\left(K_{i j}\right)=0$. Очевидно, что $S H_{N}(\nu)=S H_{1}(0) \otimes$ $S H_{N}^{\prime}(\nu)$, где $S H_{1}(0)$ порождена образующими

$$
a^{\alpha}=\sum_{i} a_{i}^{\alpha} / \sqrt{N}
$$

удовлетворяюшими соотношениям $\left[a^{\alpha}, a^{\beta}\right]=\epsilon^{\alpha \beta}$, а $S H_{N}^{\prime}(\nu)$ порождена перестановками $K_{i j}$ и линейными комбинациями вида $\sum_{i} \lambda_{i} a_{i}^{\alpha}$, в которых коэффициенты $\lambda_{i}$ удовлетворяют условиям $\sum_{i} \lambda_{i}=0$.

$S H_{N}^{\prime}(\nu)$ содержит в качестве подалгебры групповую алгебру $\mathbb{C}\left[S_{N}\right]$ группы подстановок $S_{N}$, порожденную элементарными подстановками $K_{i j}$. На волновых функциях модели Калоджеро (1) группа $S_{N}$ действует тривиально, однако для любого ее нетривиального представления сушествует матричная версия модели Калоджеро, в которой реализуется это представление [8].

Суперследом на произвольной ассоциативной супералгебре $\mathcal{A}$ называется комплекснозначная линейная функция $\operatorname{str}(\cdot)$ на $\mathcal{A}$, удовлетворяюшая условию

$$
\operatorname{str}(f g)=(-1)^{\pi(f) \pi(g)} \operatorname{str}(g f)
$$


для любых элементов $f, g \in \mathcal{A}$ с определенной четностью. В [9] доказано, что супералгебры $S H_{N}(\nu)$ имеют нетривиальные суперследы и что размерность пространства суперследов на $S H_{N}(\nu)$ (количество суперследов) равна числу различных представлений числа $N$ в виде суммы положительных нечетных целых чисел. В частности, на $S H_{2}(\nu)$ имеется только один суперслед, а на $S H_{3}(\nu)$ - два различных суперследа. Поскольку $S H_{N}(\nu)=S H_{1}(0) \otimes S H_{N}^{\prime}(\nu)$ и $S H_{1}(0)$ имеет один суперслед, то $S H_{N}(\nu)$ и $S H_{N}^{\prime}(\nu)$ имеют одинаковое число суперследов.

Знание суперследов на $S H_{N}(\nu)$ полезно в различных отношениях. Важно, что они определяют полилинейные инвариантные формы

$$
\operatorname{str}\left(f_{1} f_{2} \ldots f_{n}\right),
$$

что позволяет, например, строить лагранжианы при работе с динамическими теориями, основанными на этих супералгебрах. В частности, каждый суперслед определяет билинейную форму

$$
B_{\mathrm{Str}}(f, g)=\operatorname{str}(f g) .
$$

Так как нуль-векторы инвариантной билинейной формы образуют очевидным образом двусторонний идеал супералгебры, знание суперследов дает инструмент для исследования таких идеалов. Так, в случае $S H_{2}^{\prime}(\nu)$, соответствуюшем обычной двухчастичной модели Калоджеро, единственный суперслед определяет билинейную форму (6), которая вырождена при полуцелых значениях $\nu[10]$, а значит, супералгебра $S H_{2}^{\prime}(\nu)$ имеет идеал при таких $\nu$. При $N \geqslant 3$ алгебры $S H_{N}(\nu)$ имеют более одного суперследа. Для конечномерных супералгебр этого было бы достаточно для существования двусторонних идеалов, однако для рассматриваемых бесконечномерных супералгебр сушествование идеалов в полной мере еще не изучено.

В настоящей работе доказано, что в $S H_{3}^{\prime}(\nu)$ при $\nu=n \pm 1 / 3$ и $\nu=n+1 / 2$ существует по одномерному пространству суперследов, у которых соответствующие билинейные формы (6) вырождены, и что при других значениях $\nu$ таких суперследов нет. Следует заметить, что если существование суперследов на рассматриваемой супералгебре зависит от выбора $\mathbb{Z}_{2}$-градуировки, то сушествование идеала от градуировки не зависит. Открытыми остались вопросы о виде фактор-алгебры (в случае $S H_{2}^{\prime}(\nu)$ при $\nu=n+1 / 2$ это $\mathrm{Mat}_{n}$ ) и о сушествовании идеалов, которые не порождены никакими суперследами.

В следуюшем разделе выписаны образуюшие и соотношения между ними в ассоциативной супералгебре $S H_{3}^{\prime}(\nu)$, затем получены и решены уравнения для производящих функций суперследов, и в последнем разделе найдены все значения $\nu$, при которых $S H_{3}^{\prime}(\nu)$ имеет идеал, порожденный суперследом. 


\section{2. СУПЕРАЛГЕБРА $S H_{3}^{\prime}(\nu)$}

Приведем образуюшие и соотношения между образуюшими в ассоциативной супералгебре $S H_{3}^{\prime}(\nu)$.

Пусть $\lambda=\exp (2 \pi i / 3)$. Выберем в качестве базиса в $\mathbb{C}\left[S_{3}\right]$ векторы

$$
\begin{aligned}
& L_{k}=\frac{1}{3}\left(\lambda^{k} K_{12}+K_{23}+\lambda^{-k} K_{31}\right), \\
& Q_{k}=\frac{1}{3}\left(1+\lambda^{k} K_{12} K_{13}+\lambda^{-k} K_{12} K_{23}\right), \\
& L_{ \pm} \stackrel{\text { def }}{=} L_{ \pm 1}, \quad Q_{ \pm} \stackrel{\text { def }}{=} Q_{ \pm 1}
\end{aligned}
$$

(индексы $k$ здесь рассматриваются по модулю 3 ), а вместо образуюших $a_{i}^{\alpha}$ - векторы

$$
\begin{array}{lll}
x^{\alpha}=a_{1}^{\alpha}+\lambda a_{2}^{\alpha}+\lambda^{2} a_{3}^{\alpha}, & x \stackrel{\text { def }}{=} x^{0}, & x^{+} \stackrel{\text { def }}{=} x^{1}, \\
y^{\alpha}=a_{1}^{\alpha}+\lambda^{2} a_{2}^{\alpha}+\lambda a_{3}^{\alpha}, & y \stackrel{\text { def }}{=} y^{0}, & y^{+} \stackrel{\text { def }}{=} y^{1},
\end{array}
$$

получаюшиеся из $a_{i}^{\alpha}$ вычитанием центра масс

$$
a^{\alpha}=\frac{1}{3} \sum_{i=1,2,3} a_{i}^{\alpha} .
$$

На супералгебре $S H_{3}^{\prime}(\nu)$ действует алгебра Ли $\mathfrak{s l}_{2}$ внутренних дифференцирований, порожденная генераторами $T^{\alpha \beta}$ :

$$
T^{\alpha \beta}=\frac{1}{3}\left(x^{\alpha} y^{\beta}+x^{\beta} y^{\alpha}\right)
$$

с коммутационными соотношениями

$$
\left[T^{\alpha \beta}, T^{\gamma \delta}\right]=\epsilon^{\alpha \gamma} T^{\beta \delta}+\epsilon^{\alpha \delta} T^{\beta \gamma}+\epsilon^{\beta \gamma} T^{\alpha \delta}+\epsilon^{\beta \delta} T^{\alpha \gamma},
$$

которая действует на образуюших элементах $x^{\alpha}$ и $y^{\alpha}$ как на $\mathfrak{s l}_{2}$-векторах:

$$
\left[T^{\alpha \beta}, x^{\gamma}\right]=\epsilon^{\alpha \gamma} x^{\beta}+\epsilon^{\beta \gamma} x^{\alpha}, \quad\left[T^{\alpha \beta}, y^{\gamma}\right]=\epsilon^{\alpha \gamma} y^{\beta}+\epsilon^{\beta \gamma} y^{\alpha}
$$

и оставляет инвариантной подалгебру $\mathbb{C}\left[S_{3}\right]:\left[T^{\alpha \beta}, K_{i j}\right]=0$.

Вся супералгебра $S H_{3}^{\prime}(\nu)$ разбивается в прямую сумму неприводимых представлений алгебры $\mathfrak{s l}_{2}$. Так как всякое подпространство пространства $S H_{3}^{\prime}(\nu)$, на котором $\mathfrak{s l}_{2}$ (7) имеет нетривиальное неприводимое представление, состоит из линейных комбинаций векторов вида $\left[f, T^{\alpha \beta}\right]$, то всякий суперслед на таком подпространстве равен нулю. 
Отличными от нуля суперследы могут быть только на $H_{3}^{0}(\nu) \subset S H_{3}^{\prime}(\nu)$ - ассоциативной подалгебре всех $\mathfrak{s l}_{2}$-синглетов.

Введем величину

$$
m=\frac{1}{4}\left\{x^{\alpha}, y_{\alpha}\right\}
$$

которая является синглетом относительно действия $\mathfrak{s l}_{2}(7)$ и может быть выражена также в виде

$$
m=\frac{1}{2}\left(x^{\alpha} y_{\alpha}+3+9 \nu L_{0}\right)
$$

или эквивалентно

$$
m=\frac{1}{2}\left(y_{\alpha} x^{\alpha}-3-9 \nu L_{0}\right)
$$

В этих формулах греческие индексы поднимаются и опускаются при помоши тензора $\epsilon^{\alpha \beta}: a^{\alpha}=\epsilon^{\alpha \beta} a_{\beta}$. Очевидно, что все остальные синглеты в $H_{3}^{0}(\nu)$ являются полиномами от этой величины $m$ с коэффициентами в $\mathbb{C}\left[S_{3}\right]$.

Введенные таким образом образуюшие $x^{\alpha}, y^{\alpha}, Q_{i}, L_{i}$ и синглет $m$ удовлетворяют следуюшим соотношениям:

$$
\begin{aligned}
& L_{i} L_{j}=\delta_{i+j} Q_{j}, \quad L_{i} Q_{j}=\delta_{i-j} L_{j}, \\
& Q_{i} L_{j}=\delta_{i+j} L_{j}, \quad Q_{i} Q_{j}=\delta_{i-j} Q_{j},
\end{aligned}
$$

где $\delta_{i}=\delta_{i 0}$

$$
\begin{aligned}
L_{i} x^{\alpha}=y^{\alpha} L_{i+1}, & L_{i} y^{\alpha}=x^{\alpha} L_{i-1}, \\
Q_{i} x^{\alpha}=x^{\alpha} Q_{i+1}, & Q_{i} y^{\alpha}=y^{\alpha} Q_{i-1}, \\
L_{i} m=-m L_{i}, & Q_{i} m=m Q_{i}, \\
{\left[x, x^{+}\right]=-9 \nu L_{+}, \quad\left[y, y^{+}\right]=-9 \nu L_{-}, } &
\end{aligned}
$$

и

$$
\begin{aligned}
& {\left[m, x^{\alpha}\right]=\frac{3}{2}\left(x^{\alpha}+3 \nu x^{\alpha} L_{0}+3 \nu L_{0} x^{\alpha}\right)} \\
& {\left[m, y^{\alpha}\right]=-\frac{3}{2}\left(y^{\alpha}+3 \nu y^{\alpha} L_{0}+3 \nu L_{0} y^{\alpha}\right)}
\end{aligned}
$$


из которых следуют соотношения

$$
\begin{aligned}
m Q_{+} x^{\alpha} Q_{-} & =Q_{+} x^{\alpha} Q_{-}\left(m+\frac{3}{2}\right) \\
m Q_{-} y^{\alpha} Q_{+} & =Q_{-} y^{\alpha} Q_{+}\left(m-\frac{3}{2}\right) \\
m Q_{-} x^{\alpha} Q_{0} & =Q_{-} x^{\alpha} Q_{0}\left(m+\frac{3}{2}+\frac{9}{2} \nu L_{0}\right) \\
m Q_{+} y^{\alpha} Q_{0} & =Q_{+} y^{\alpha} Q_{0}\left(m-\frac{3}{2}-\frac{9}{2} \nu L_{0}\right) \\
Q_{0} x^{\alpha} Q_{+} m & =\left(m-\frac{3}{2}-\frac{9}{2} \nu L_{0}\right) Q_{0} x^{\alpha} Q_{+} \\
Q_{0} y^{\alpha} Q_{-} m & =\left(m+\frac{3}{2}+\frac{9}{2} \nu L_{0}\right) Q_{0} y^{\alpha} Q_{-}
\end{aligned}
$$

Как показано в [9], всякий суперслед на $S H_{3}^{\prime}(\nu)$ полностью определяется его значениями на $\mathbb{C}\left[S_{3}\right] \subset S H_{3}^{\prime}(\nu)$, т.е. значениями $\operatorname{str}(1), \operatorname{str}\left(K_{12}\right)=\operatorname{str}\left(K_{23}\right)=\operatorname{str}\left(K_{31}\right)$ и $\operatorname{str}\left(K_{12} K_{23}\right)=\operatorname{str}\left(K_{12} K_{13}\right)$. При этом условие (5) является непротиворечивым и допускает продолжение суперследа с $\mathbb{C}\left[S_{3}\right]$ на всю $S H_{3}^{\prime}(\nu)$, только если

$$
\operatorname{str}\left(K_{i j}\right)=\nu\left(-2 \operatorname{str}(1)-\operatorname{str}\left(K_{12} K_{23}\right)\right) .
$$

Поскольку значение всякого суперследа может быть отличным от нуля только на $\mathfrak{s l}_{2}$-синглетных элементах супералгебры, то достаточно найти суперследы лишь на $H_{3}^{0}(\nu)$.

\section{3. ПРОИЗВОДЯЩИЕ ФУНКЦИИ}

В этом разделе получены и решены уравнения для производящих функций суперследов.

Введем производящие функиии:

$$
\begin{aligned}
& F(\xi)=\operatorname{str}\left(\operatorname{sh}\left(\frac{2}{3} \xi m\right)\left(Q_{+}-Q_{-}\right)\right), \\
& \Phi(\xi)=\operatorname{str}\left(\operatorname{ch}\left(\frac{2}{3} \xi m\right)\left(Q_{+}+Q_{-}\right)\right), \\
& \Psi(\xi)=\operatorname{str}\left(\operatorname{ch}\left(\xi \sqrt{\frac{4}{9} m^{2}+9 \nu^{2}}\right) Q_{0}\right),
\end{aligned}
$$

которые полностью описывают суперследы на $S H_{3}^{\prime}(\nu)$. Действительно, из (16) и (5) следует, что при $k>0$ имеют место равенства $\operatorname{str}\left(m^{k} L_{i}\right)=\operatorname{str}\left(m^{k-1} L_{i} m\right)=-\operatorname{str}\left(m^{k} L_{i}\right)$, поэтому $\operatorname{str}\left(f(m) L_{i}\right)=f(0) \operatorname{str}\left(L_{i}\right)$, а т.к. $\operatorname{str}\left(m^{k} Q_{0}\right)=\operatorname{str}\left(m^{k} L_{0}^{2}\right)=\operatorname{str}\left(L_{0} m^{k} L_{0}\right)=$ $(-1)^{k} \operatorname{str}\left(m^{k} L_{0}^{2}\right), \operatorname{то} \operatorname{str}\left(\operatorname{sh}(\xi m) Q_{0}\right)=0$. Аналогично

$$
\operatorname{str}\left(\operatorname{sh}\left(\frac{2}{3} \xi m\right)\left(Q_{+}+Q_{-}\right)\right)=0 \text { и } \operatorname{str}\left(\operatorname{ch}\left(\frac{2}{3} \xi m\right)\left(Q_{+}-Q_{-}\right)\right)=0 .
$$


Уравнения для производящих функций. Два уравнения для производяшей функции суперследов можно получить дифференцированием определения (26) и два дифференцированием (27). Для получения первого уравнения надо использовать выражение (10) для $m$ в члене $m Q_{+}$и $(11)$ в члене $m Q_{-}$. Для получения второго уравнения следует использовать $(11)$ для $m$ в члене $m Q_{+}$и $(10)$ в члене $m Q_{-}$. Первое уравнение имеет следующий вид:

$$
F^{\prime}(\xi)=\frac{2}{3} \operatorname{str}\left(\operatorname{ch}\left(\frac{2}{3} \xi m\right)\left(\frac{1}{2}\left(x^{\alpha} y_{\alpha}+3+9 \nu L_{0}\right) Q_{+}-\frac{1}{2}\left(y_{\alpha} x^{\alpha}-3-9 \nu L_{0}\right) Q_{-}\right)\right)
$$

и при помощи $(27),(12),(15),(19),(20)$ и общих свойств суперследов (5) может быть сведено к виду

$$
F^{\prime}(\xi)=\Phi(\xi)-\frac{1}{3} \operatorname{str}\left(\operatorname{ch}\left(\frac{2}{3} \xi m+\xi\right) y_{\alpha} x^{\alpha} Q_{-}-\operatorname{ch}\left(\frac{2}{3} \xi m-\xi\right) x^{\alpha} y_{\alpha} Q_{+}\right)
$$

Подставив в (29) вместо $x^{\alpha} y_{\alpha}$ и $y_{\alpha} x^{\alpha}$ их выражения через $m$ из (10) и (11) и разложив выражения

$$
\operatorname{ch}\left(\frac{2}{3} \xi m \pm \xi\right)
$$

легко получить искомое уравнение

$$
\Phi=F^{\prime}+(\operatorname{sh} \xi \Phi)^{\prime}-(\operatorname{ch} \xi F)^{\prime} .
$$

Аналогично выводится второе уравнение:

$$
F^{\prime}(\xi)=\frac{2}{3} \operatorname{str}\left(\operatorname{ch}\left(\frac{2}{3} \xi m\right)\left(\frac{1}{2}\left(y_{\alpha} x^{\alpha}-3-9 \nu L_{0}\right) Q_{+}-\frac{1}{2}\left(x^{\alpha} y_{\alpha}+3+9 \nu L_{0}\right) Q_{-}\right)\right)
$$

из которого при помощи $(22),(21),(12)$ и общих свойств суперследов $(5)$ легко получить

$$
\begin{aligned}
F^{\prime}(\xi)= & -\Phi(\xi)-\frac{1}{3} \operatorname{str}\left(\operatorname{ch}\left(\frac{2}{3} \xi m-\xi-3 \nu \xi L_{0}\right) x^{\alpha} y_{\alpha} Q_{0}-\right. \\
& \left.-\operatorname{ch}\left(\frac{2}{3} \xi m+\xi+3 \nu \xi L_{0}\right) y_{\alpha} x^{\alpha} Q_{0}\right)= \\
= & -\Phi-\frac{1}{3} \operatorname{str}\left(\operatorname{ch} \xi \operatorname{ch}\left(\frac{2}{3} \xi m-3 \nu \xi L_{0}\right)\left(2 m-3-9 \nu L_{0}\right) Q_{0}-\right. \\
& -\operatorname{sh} \xi \operatorname{sh}\left(\frac{2}{3} \xi m-3 \nu \xi L_{0}\right)\left(2 m-3-9 \nu L_{0}\right) Q_{0}- \\
& -\operatorname{ch} \xi \operatorname{ch}\left(\frac{2}{3} \xi m+3 \nu \xi L_{0}\right)\left(2 m+3+9 \nu L_{0}\right) Q_{0}- \\
& \left.-\operatorname{sh} \xi \operatorname{sh}\left(\frac{2}{3} \xi m+3 \nu \xi L_{0}\right)\left(2 m+3+9 \nu L_{0}\right) Q_{0}\right)
\end{aligned}
$$


Из соотношения $m L_{0}=-L_{0} m$ следует, что

$$
\begin{aligned}
& \operatorname{ch}\left(\alpha m+\beta L_{0}\right) Q_{0}=\left(\operatorname{ch} \sqrt{\alpha^{2} m^{2}+\beta^{2}}\right) Q_{0}, \\
& \operatorname{sh}\left(\alpha m+\beta L_{0}\right) Q_{0}=\frac{1}{\sqrt{\alpha^{2} m^{2}+\beta^{2}}}\left(\operatorname{sh} \sqrt{\alpha^{2} m^{2}+\beta^{2}}\right)\left(\alpha m+\beta L_{0}\right) Q_{0},
\end{aligned}
$$

что позволяет привести уравнение (31) к следующему виду:

$$
F^{\prime}=-\Phi+2\left(\operatorname{sh} \xi \operatorname{str}\left(\operatorname{ch} \sqrt{\left(\frac{2}{3} \xi m\right)^{2}+(3 \nu \xi)^{2}}\right) Q_{0}\right)^{\prime}+2\left(\operatorname{ch} \xi \operatorname{sh}(3 \nu \xi) \operatorname{str} L_{0}\right)^{\prime}
$$

Аналогично выводится вторая пара уравнений.

Окончательно получается система уравнений

$$
\begin{aligned}
F^{\prime} & =-\Phi+2(\operatorname{sh} \xi \Psi)^{\prime}+2\left(\operatorname{ch} \xi \operatorname{sh}(3 \nu \xi) \operatorname{str} L_{0}\right)^{\prime} \\
\Phi^{\prime} & =-F-2(\operatorname{ch} \xi \Psi)^{\prime}-2\left(\operatorname{sh} \xi \operatorname{sh}(3 \nu \xi) \operatorname{str} L_{0}\right)^{\prime} \\
\Phi & =F^{\prime}+(\operatorname{sh} \xi \Phi)^{\prime}-(\operatorname{ch} \xi F)^{\prime} \\
F & =\Phi^{\prime}+(\operatorname{ch} \xi \Phi)^{\prime}-(\operatorname{sh} \xi F)^{\prime}
\end{aligned}
$$

в которой уравнения (34) и (35) эквивалентны, со следующими начальными условиями:

$$
\begin{aligned}
& F(0)=0, \\
& \Phi(0)=\operatorname{str}\left(Q_{+}+Q_{-}\right), \\
& \Psi(0)=\operatorname{str}\left(Q_{0}\right) .
\end{aligned}
$$

Для этой системы уравнений можно легко найти три интеграла движения. Чтобы найти первый, достаточно вычесть (34) из (32), чтобы найти второй, следует вычесть (35) из (33), и для того чтобы найти третий, достаточно рассмотреть линейную комбинацию $\operatorname{sh} \xi \times($ уравнение $(32))+\operatorname{ch} \xi \times($ уравнение $(33))$.

Результат всех этих действий имеет вид

$$
\begin{aligned}
(F(2-\operatorname{ch} \xi)+\Phi \operatorname{sh} \xi+\Psi(-2 \operatorname{sh} \xi))^{\prime} & =2\left(\operatorname{ch} \xi \operatorname{sh}(3 \nu \xi) \operatorname{str}\left(L_{0}\right)\right)^{\prime} \\
(F(-\operatorname{sh} \xi)+\Phi(2+\operatorname{ch} \xi)+\Psi(2 \operatorname{ch} \xi))^{\prime} & =-2\left(\operatorname{sh} \xi \operatorname{sh}(3 \nu \xi) \operatorname{str}\left(L_{0}\right)\right)^{\prime} \\
(F \operatorname{sh} \xi+\Phi \operatorname{ch} \xi+2 \Psi)^{\prime} & =-2 \operatorname{sh}(3 \nu \xi) \operatorname{str}\left(L_{0}\right)
\end{aligned}
$$

5 Теоретическая и математическая физика, т. 116, № 1, 1998 г. 
Решение. Решение полученной системы уравнений в терминах производящих функций, более удобных для дальнейшего рассмотрения, выглядит следующим образом:

$$
\mathcal{Q}_{+} \stackrel{\text { def }}{=} \operatorname{str}\left(e^{(2 / 3) \xi m} Q_{+}\right)=\frac{P_{+}}{\Delta}
$$

где

$$
\begin{aligned}
P_{+}= & 2 \nu S_{1}\left(\frac{\operatorname{ch}(3 \nu \xi)}{3 \nu}\left(-e^{2 \xi}+2 e^{-\xi}\right)+\operatorname{sh}(3 \nu \xi)\left(e^{2 \xi}+e^{-\xi}\right)\right)+ \\
& +\frac{S_{2}}{2}\left(e^{-2 \xi}-2 e^{\xi}+3\right) \\
\mathcal{Q}_{-} \stackrel{\text { def }}{=} & \operatorname{str}\left(e^{(2 / 3) \xi m} Q_{-}\right)=\frac{P_{-}}{\Delta}
\end{aligned}
$$

где

$$
\begin{aligned}
P_{-}= & 2 \nu S_{1}\left(\frac{\operatorname{ch}(3 \nu \xi)}{3 \nu}\left(-e^{-2 \xi}+2 e^{\xi}\right)-\operatorname{sh}(3 \nu \xi)\left(e^{-2 \xi}+e^{\xi}\right)\right)+ \\
& +\frac{S_{2}}{2}\left(e^{2 \xi}-2 e^{-\xi}+3\right) \\
\mathcal{Q}_{0}^{ \pm} \stackrel{\text { def }}{=} & \operatorname{str}\left(\operatorname{ch}\left(\xi \sqrt{\frac{4}{9} m^{2}+9 \nu^{2}}\left(Q_{0} \pm L_{0}\right)\right)\right)=\frac{P_{0}}{\Delta} \pm \nu S_{1} \operatorname{ch}(3 \nu \xi),
\end{aligned}
$$

где

$$
\begin{aligned}
& P_{0}= 2 \nu S_{1}\left(-3 \frac{\operatorname{ch}(3 \nu \xi)}{3 \nu}+\frac{\operatorname{sh}(3 \nu \xi)}{2}\left(-e^{3 \xi}+e^{-3 \xi}\right)\right)+ \\
& \quad+\frac{S_{2}}{2}\left(e^{2 \xi}+e^{-2 \xi}-2 e^{\xi}-2 e^{-\xi}\right) ; \\
& \mathcal{R}_{0} \stackrel{\text { def }}{=} \operatorname{str}\left(\operatorname{sh}(\xi m) Q_{0}\right)=0 ; \\
& \mathcal{L}_{ \pm} \stackrel{\text { def }}{=} \operatorname{str}\left(e^{(2 / 3) \xi m} L_{ \pm 1}\right)=0 ; \\
& \mathcal{L}_{0} \stackrel{\text { def }}{=} \operatorname{str}\left(e^{(2 / 3) \xi m} L_{0}\right)=\nu S_{1} .
\end{aligned}
$$

Здесь $\Delta=\exp (-3 \xi)(\exp (3 \xi)+1)^{2}$, а $S_{1}$ и $S_{2}$ - произвольные параметры, определяюшие суперслед в двумерном пространстве суперследов:

$$
S_{1}=-2 \operatorname{str}(1)-\operatorname{str}\left(K_{12} K_{23}\right), \quad S_{2}=\frac{8}{3} \operatorname{str}(1)-\frac{2}{3} \operatorname{str}\left(K_{12} K_{23}\right)
$$




\section{4. ИДЕАЛЫ}

Легко показать, что если некоторый идеал $\mathcal{I} \subset S H_{3}^{\prime}(\nu)$ имеет ненулевое пересечение с $H_{3}^{0}$ (которое является идеалом в $H_{3}^{0}$ ), то $\mathcal{I}$ обладает ненулевым пересечением с одним из подпространств в $H_{3}^{0}$, состоящих из элементов вида $\left\{Q_{ \pm} f(m)\right\}$ (случаи $P_{ \pm}$), либо с одним из подпространств, состоящих из элементов вида $\left\{\left(Q_{0} \pm L_{0}\right) f(m)\right\}$ (случай $P_{0}$ ).

Сначала будем искать элементы идеала вида $\left\{Q_{+} f(m)\right\}$, т.е. такие полиномиальные функции $f$, что

$$
f\left(\frac{\mathrm{d}}{\mathrm{d} \xi}\right) \mathcal{Q}_{+}=0
$$

Если хоть одна такая функция существует, то (36) есть линейная комбинация экспонент

$$
\mathcal{Q}_{+}=\sum_{i} p_{i} e^{b_{i} \xi}
$$

где $p_{i}$ - некоторые полиномы по переменной $\xi$.

Из (36) следует, что $p_{i}$ - на самом деле константы и что такое представление сушествует тогда и только тогда, когда все нули функции $\Delta(\xi)$ являются нулями функции $P_{+}(\xi)$ с учетом их кратностей, т.е.

$$
\begin{aligned}
P_{+}\left(\frac{1}{3}(2 k+1) \pi i\right) & =0 \quad \forall k \in \mathbb{Z} \\
P_{+}{ }^{\prime}\left(\frac{1}{3}(2 k+1) \pi i\right) & =0 \quad \forall k \in \mathbb{Z} .
\end{aligned}
$$

Второе из этих условий возникает вследствие того, что все нули функции $\Delta$ двойные.

Оказывается, условия (40) удовлетворяются тож дественно при всех значениях величин $S_{1}, S_{2}$ и $\nu$. Условия (39) ведут к системе уравнений

$$
\frac{S_{1}}{3} \cos ((2 k+1) \pi \nu)+\frac{S_{2}}{2} \cos \left(\frac{1}{3}(2 k+1) \pi\right)=0,
$$

которая имеет нетривиальные решения для $S_{1}$ и $S_{2}$ тогда и только тогда, когда при произвольных целых значениях $k$ и $l$ величина $\nu$ удовлетворяет уравнению

$$
\cos ((2 k+1) \pi \nu) \cos \left(\frac{1}{3}(2 l+1) \pi\right)=\cos ((2 l+1) \pi \nu) \cos \left(\frac{1}{3}(2 k+1) \pi\right) .
$$

Случай $l=3, \quad k=2$ дает $\cos (7 \pi \nu)-\cos (5 \pi \nu)=0$ и, следовательно, $\nu=s / 6$, где $s-$ некоторое целое число. Для того чтобы определить $s$, рассмотрим два случая: $k, l$ и $k+3, l$. Следствием этих двух случаев является соотношение

$$
(\cos (s \pi)-1) \cos \left(\frac{1}{6}(2 k+1) s \pi\right)=0 \quad \forall k,
$$


откуда следует, что либо $s=2 n$, либо $\forall k \in \mathbb{Z} \exists n \in \mathbb{Z}$ такое, что $(2 k+1) s=6 n+3$, т.е. $s=6 l+3$ с некоторым целым $l$.

Окончательно получаем, что подпространство идеала, состояшее из элементов вида $\left\{Q_{+} f(m)\right\}$ (случай $\left.P_{+}\right)$, нетривиально тогда и только тогда, когда

$$
\nu=n \pm \frac{1}{3}, \quad \frac{S_{1}}{3}=-(-1)^{n} \frac{S_{2}}{2} \text { или } \quad \nu=n+\frac{1}{2}, \quad S_{2}=0
$$

с целым значением $n$.

Случаи $P_{-}$и $P_{0}$ дают в точности эти же значения $\nu, S_{1}$ и $S_{2}$.

Другие значения $\nu$. Выше было доказано, что подалгебра синглетов $H_{3}^{0}(\nu) \subset S H_{3}^{\prime}(\nu)$ содержит идеал, порожденньй суперследом, тогда и только тогда, когда $\nu=n \pm 1 / 3$ или $\nu=n+1 / 2$. Если $H_{3}^{0}(\nu)$ содержит такой идеал, то он очевидным образом продолжается до (собственного) идеала в $S H_{3}^{\prime}(\nu)$. Здесь будет доказано, что если все билинейные формы вида $\operatorname{str}(f \cdot g)$ невырождены на $H_{3}^{0}(\nu)$, то они невырождены и на всей супералгебре $S H_{3}^{\prime}(\nu)$, т.е. что при прочих значениях $\nu$ в супералгебре $S H_{3}^{\prime}(\nu)$ нет идеалов, порожденных суперследами.

Для этого достаточно доказать, что нетривиальных решений нет ни у одной из систем уравнений

$$
\sum_{j=0}^{n} M_{i j}^{n} p_{j}=0, \quad i=1, \ldots, n,
$$

на $p_{j} \in H_{3}^{0}(\nu)$, в которых

$$
M_{i j}^{n}=\left(x^{i} y^{n-i}\left(x^{+}\right)^{n-j}\left(y^{+}\right)^{j}\right)_{0}
$$

а операция $(f)_{0}$ обозначает выделение синглетной части из полинома $f$.

Очевидно, что элементы матрицы $M^{n}$ - это полиномы от $m$ степени не выше $n$ с коэффициентами из $\mathbb{C}\left[S_{3}\right]$ :

$$
M_{i j}^{n}=\sum_{k=0}^{n} c_{i j}^{k} m^{k}
$$

причем $c_{i j}^{n} \in \mathbb{C}$. Очевидно также, что из невырожденности матрицы $\widetilde{M}^{n}$ с элементами $\left(\widetilde{M}^{n}\right)_{i j}=c_{i j}^{n}$ следует отсутствие нетривиальных решений у уравнения (43).

Найдем значения $c_{i j}^{n}$. Так как $m^{n}$ с точностью до полиномов от $x$ и $y$ меньшей степени есть линейная комбинация мономов вида $x^{i} y^{n-i}\left(x^{+}\right)^{n-i}\left(y^{+}\right)^{i}$, то $c_{i j}^{n}=0$ при $i \neq j$. Для нахождения $c_{i i}^{n}$ заметим, что

$$
x y^{+} \simeq \frac{3}{2} T^{01}-m, \quad x^{+} y \simeq \frac{3}{2} T^{01}+m,
$$


где знак $\simeq$ использован для обозначения равенства с точностью до полиномов меньшей степени, и поэтому

$$
M_{i i}^{n} \simeq\left(\frac{3}{2}\right)^{n}\left(\left(T^{01}-\frac{2}{3} m\right)^{i}\left(T^{01}+\frac{2}{3} m\right)^{n-i}\right)_{0}
$$

Ниже будет доказано, что если $f\left(m, T^{01}\right)$ - произвольный полином, то

$$
\left(f\left(m, T^{01}\right)\right)_{0} \simeq \frac{1}{2} \int_{0}^{1}\left(f\left(m, \frac{2}{3} m \tau\right)+f\left(m,-\frac{2}{3} m \tau\right)\right) d \tau .
$$

Из (45) немедленно следует, что

$$
\begin{aligned}
M_{i i}^{n} & \simeq \frac{1}{2}\left(\frac{2}{3} m\right)^{n} \int_{0}^{1}\left((\tau-1)^{i}(\tau+1)^{n-i}+(-\tau-1)^{i}(-\tau+1)^{n-i}\right) d \tau= \\
& =\frac{(-1)^{i}}{2}\left(\frac{2}{3} m\right)^{n} \int_{0}^{1}\left((1-\tau)^{i}(1+\tau)^{n-i}+(1+\tau)^{i}(1-\tau)^{n-i}\right) d \tau
\end{aligned}
$$

и, следовательно, $c_{i i}^{n} \neq 0(i=0, \ldots, n)$, что вместе с $c_{i j}^{n}=0$ при $i \neq j$ приводит к невырожденности $\widetilde{M}$, т.е. к тому, что у уравнений (43) нет нетривиальных решений.

Для доказательства (45) достаточно рассмотреть случай $f\left(m, T^{01}\right)=t^{k}$, где для удобства вместо выражения $T^{01}$ использована буква $t$.

Из цепочки очевидных равенств

$$
\begin{aligned}
0 & =\left(\left[T^{11},\left[T^{00}, t^{k}\right]\right]\right)_{0} \simeq\left(\left[T^{11}, 2 k T^{00} t^{k-1}\right]\right)_{0} \simeq \\
& \simeq\left(-4 k(k-1) t^{k-2} T^{00} T^{11}-8 k t^{k}\right)_{0} \simeq\left(-4 k(k-1) t^{k-2}\left(9 t^{2}-4 m^{2}\right)-8 k t^{k}\right)_{0}
\end{aligned}
$$

следует

$$
\left(t^{k}\right)_{0} \simeq \frac{4}{9} m^{2} \frac{k-1}{k+1}\left(t^{k-2}\right)_{0} \simeq \begin{cases}\frac{1}{k+1}\left(\frac{2}{3} m\right)^{k}=\int_{0}^{1}\left(\frac{2}{3} m \tau\right)^{k} d \tau & \text { при четных } k, \\ 0 & \text { при нечетных } k,\end{cases}
$$

что завершает доказательство.

Автор благодарен М. А. Васильеву за полезные обсуждения.

Настоящая работа была поддержана Российским фондом фундаментальных исследований, гранты № 96-01-01144 и № 96-15-96463.

\section{Список литературы}

[1] F. Calogero. J. Math. Phys. 1969. V. 10. P. 2191, 2197; 1971. V. 12. P. 419.

[2] M. A. Olshanetsky, A. M. Perelomov. Phys. Rep. 1983. V. 94. P. 313.

[3] A. Polychronakos. Phys. Rev. Lett. 1992. V. 69. P. 703.

[4] L. Brink, H. Hansson, M. A. Vasiliev. Phys. Lett. B. 1992. V. 286. P. 109.

[5] C. F. Dunkl. Trans. Am. Math. Soc. 1989. V. 311. P. 167.

[6] L. Brink, H. Hansson, S. E. Konstein, M. A. Vasiliev. Nucl. Phys. B. 1993. V. 401. P. 591.

[7] А.М. Переломов. ТМФ. 1971. Т. 6. С. 364.

[8] М. А. Васильев, О. В. Додлов, С. Е. Конштейн. Письма в ЖЭТФ. 1993. Т. 58. С. 911.

[9] S. E. Konstein, M. A. Vasiliev. J. Math. Phys. 1996. V. 37. P. 2872.

[10] М. А. Васильев. Письма в ЖЭТФ. 1989. Т. 50. В. 8. С. 344; M. A. Vasiliev. Int. J. Mod. Phys. A. 1991. V. 6. P. 1115. 